\title{
Homeomorphism groups of Sierpiński carpets and Erdös space
}

by

\author{
Jan J. Dijkstra and Dave Visser (Amsterdam)
}

\begin{abstract}
Erdős space $\mathfrak{E}$ is the "rational" Hilbert space, that is, the set of vectors in $\ell^{2}$ with all coordinates rational. Erdős proved that $\mathfrak{E}$ is one-dimensional and homeomorphic to its own square $\mathfrak{E} \times \mathfrak{E}$, which makes it an important example in dimension theory. Dijkstra and van Mill found topological characterizations of $\mathfrak{E}$. Let $M_{n}^{n+1}, n \in \mathbb{N}$, be the $n$-dimensional Menger continuum in $\mathbb{R}^{n+1}$, also known as the $n$-dimensional Sierpiński carpet, and let $D$ be a countable dense subset of $M_{n}^{n+1}$. We consider the topological group $\mathcal{H}\left(M_{n}^{n+1}, D\right)$ of all autohomeomorphisms of $M_{n}^{n+1}$ that map $D$ onto itself, equipped with the compact-open topology. We show that under some conditions on $D$ the space $\mathcal{H}\left(M_{n}^{n+1}, D\right)$ is homeomorphic to $\mathfrak{E}$ for $n \in \mathbb{N} \backslash\{3\}$.
\end{abstract}

1. Introduction. All spaces in this paper are assumed to be separable and metrizable. If $X$ is locally compact then we equip the group $\mathcal{H}(X)$ of homeomorphisms of $X$ with the compact-open topology. If $A$ is a subset of $X$ then $\mathcal{H}(X, A)$ stands for the subgroup $\{h \in \mathcal{H}(X): h(A)=A\}$ of $\mathcal{H}(X)$.

Let $D$ be a countable dense subset of a locally compact space $X$. In [5] Dijkstra and van Mill show that if $X$ contains a nonempty open subset homeomorphic to $\mathbb{R}^{n}$ for $n \geq 2$, to an open subset of the Hilbert cube, or to an open subset of some universal Menger continuum $\mu^{n}$ for $n \in \mathbb{N}$, then $\mathcal{H}(X, D)$ is homeomorphic to $\mathfrak{E}$. In line with these results we consider in this paper the topological group $\mathcal{H}\left(M_{n}^{n+1}, D\right)$ for $n \in \mathbb{N}$. Here $M_{n}^{n+1}$ is the $n$-dimensional Menger continuum in $\mathbb{R}^{n+1}$ (see Engelking [6, §1.11]), also known as the $n$-dimensional Sierpiński carpet, and $D$ is a countable dense subset of $M_{n}^{n+1}$. In our main result, Theorem 3.1, we show that under some conditions on $D$ the space $\mathcal{H}\left(M_{n}^{n+1}, D\right)$ is homeomorphic to $\mathfrak{E}$ for $n \in \mathbb{N} \backslash\{3\}$. The proof is based on the proof of [5, Theorem 10.4] where

2010 Mathematics Subject Classification: Primary 57S05.

Key words and phrases: homeomorphism group, Sierpiński carpet, Erdős space, almost zero-dimensional. 
Dijkstra and van Mill use their characterization of $\mathfrak{E}$ to deal with the $\mu^{n}$ case. We also heavily rely on Dijkstra [4, §5] where it is shown that there are closed imbeddings of Erdős-type subspaces of $\ell^{1}$ (see Theorem 2.14) in $\mathcal{H}\left(M_{n}^{n+1}\right.$ ) if $n \in \mathbb{N} \backslash\{3\}$. The main complication is that $M_{n}^{n+1}$, in contrast to the $n$-dimensional universal Menger continuum considered in [5, Theorem 10.4], is not homogeneous.

2. Preliminaries. Let $\mathbb{R}^{+}=[0, \infty)$. We shall use a number of compactifications of $\mathbb{R}^{m}$. Let $S^{m}$ denote the one-point compactification of $\mathbb{R}^{m}$. We let $\hat{\mathbb{R}}$ denote the compactification $[-\infty, \infty]$ of $\mathbb{R}$. We shall use the convention that $\pm \infty+t= \pm \infty$ when $t \in \mathbb{R}$. This extends the addition operation on $\mathbb{R}^{m}$ to a continuous function from $\hat{\mathbb{R}}^{m} \times \mathbb{R}^{m}$ to $\hat{\mathbb{R}}^{m}$. An $m$-cell is any space that is homeomorphic to $I^{m}$, where $I=[0,1]$. For a set $A$ in a topological space we let $\partial A$ denote the boundary of $A$ and $\operatorname{Int}(A)$ the interior of $A$.

Recall that for a compact space $X$ the compact-open topology on $\mathcal{H}(X)$ coincides with the topology of uniform convergence. We denote the identity element of $\mathcal{H}(X)$ by $e_{X}$. If $O$ is an open subset of $X$ then we say that $h \in \mathcal{H}(X)$ is supported on $O$ if $h$ is equal to the identity on $X \backslash O$, i.e. if $h \uparrow(X \backslash O)=e_{X \backslash O}$. We write $\mathcal{H}_{O}(X)$ for the subgroup of $\mathcal{H}(X)$ consisting of all homeomorphisms of $X$ that are supported on $O$, so $\mathcal{H}_{O}(X)=\{h \in$ $\left.\mathcal{H}(X): h \uparrow(X \backslash O)=e_{X \backslash O}\right\}$. Furthermore, we let $\mathcal{H}_{O}(X, A)$ stand for the subgroup $\mathcal{H}_{O}(X) \cap \mathcal{H}(X, A)$ of $\mathcal{H}(X)$.

We need the following elementary result; see [5, Lemma 10.3].

Lemma 2.1. Let $f: X \rightarrow Y$ and $g: Y \rightarrow Z$ be continuous. If $g \circ f$ is a closed imbedding then so is $f$.

We give the definition of an $n$-dimensional Sierpiński carpet.

Definition 2.2. Let $n \in \mathbb{N}$. A nowhere dense subset $X$ of $S^{n+1}$ is called an $n$-dimensional Sierpinski carpet if the collection $\left\{U_{i}: i \in \mathbb{N}\right\}$ of components of $S^{n+1} \backslash X$ forms a null sequence such that the closures of the $U_{i}$ 's are a pairwise disjoint collection and every $S^{n+1} \backslash U_{i}$ is an $(n+1)$-cell.

The Menger continuum $M_{n}^{n+1}$, constructed according to the "middle third" method (see Engelking [6, §1.11]) is a standard example of an $n$ dimensional Sierpiński carpet. The following characterization theorem is due to Whyburn [11] (for $n=1$ ) and Cannon [2] (for $n \geq 2$ ).

TheOREm 2.3. Let $X$ and $Y$ be two $n$-dimensional Sierpinski carpets for $n \in \mathbb{N} \backslash\{3\}$ and let $U$ and $V$ be components of $S^{n+1} \backslash X$, respectively $S^{n+1} \backslash Y$. If $h$ is a homeomorphism from the boundary of $U$ to the boundary of $V$, then $h$ can be extended to a homeomorphism from $X$ to $Y$.

REMARK 2.4. In Theorem 2.3, let $S$ and $T$ be components of $S^{n+1} \backslash X$, respectively $S^{n+1} \backslash Y$, such that $S \neq U$ and $T \neq V$. The proofs of Lemma 1 
and Theorem 1 in [2 together with the Annulus Theorem ([2]), which enables one to control where the boundary of a component of $S^{n+1} \backslash X$ is mapped to, imply that we can extend $h$ to a homeomorphism $\bar{h}: X \rightarrow Y$ in such a way that $\bar{h}(\partial S)=\partial T$.

Definition 2.5. A point $x$ of an $n$-dimensional Sierpiński carpet $X$ is called a boundary point of $X$ if it lies on a nonseparating copy $S$ of $S^{n}$ in $X$, that is, $X \backslash S$ is connected. If $x$ is not a boundary point we call it an interior point of $X$.

Using the notation of Definition 2.2, it follows easily from Brouwer's Invariance of Domain [8, Theorem 3.6.8] and the generalized Jordan Curve Theorem [9, Theorem 36.3] that $x$ is a boundary point of $X$ if and only if $x \in \bigcup_{i=1}^{\infty} \partial U_{i}$ and that every $\partial U_{i}$ is homeomorphic to $S^{n}$. Note that these definitions of boundary point and interior point of $X$ do not coincide with the usual meaning of these notions since $\operatorname{Int}(X)=\emptyset$. Boundary points and interior points are two topologically different types of points in $X$, both of which are represented in $X$. This means that $X$ is not homogeneous. It is well known that these points are topologically the only two different types of points in $X$ if $\operatorname{dim} X \neq 3$ (cf. Theorem 2.3 and Lemma 2.7).

Lemma 2.6. Let $n \in \mathbb{N} \backslash\{3\}$ and suppose that $x \in \partial U$, where $U$ is a component of $S^{n+1} \backslash M_{n}^{n+1}$. Then there is a local basis $\mathcal{B}_{x}$ at $x$ such that for every $B \in \mathcal{B}_{x}$ and every $y \in B \cap \partial U$ there is a homeomorphism $h$ of $M_{n}^{n+1}$ with $h(x)=y$ that is supported on $B$.

Proof. Note that it follows from Theorem 2.3 and the homogeneity of $S^{n}$ that all boundary points of $M_{n}^{n+1}$ are topologically equivalent. Therefore, it is enough to consider the boundary point $x=(0, \ldots, 0) \in \partial\left(I^{n+1}\right)$, where $\partial\left(I^{n+1}\right)$ is the boundary of the unbounded component of $\mathbb{R}^{n+1} \backslash M_{n}^{n+1}$. For $\mathcal{B}_{x}$ we take the collection $\left\{B_{i}: i \in \omega\right\}$, where $B_{i}=M_{n}^{n+1} \cap\left[0,3^{-i}\right)^{n+1}$. Now take $i \in \omega$ and a point $y \in B_{i} \cap \partial I^{n+1}$. If $y=x$ then the identity map obviously satisfies the requirements of the lemma, so we suppose that $y \neq x$. The closure of $B_{i}$ in $M_{n}^{n+1}$ is $C_{i}=M_{n}^{n+1} \cap\left[0,3^{-i}\right]^{n+1}$, so $C_{i}=3^{-i} M_{n}^{n+1}$, which means that $C_{i}$ is again an $n$-dimensional Sierpiński carpet. Note that $D_{i}=\partial\left(\left[0,3^{-i}\right]^{n+1}\right)$ is the boundary of the unbounded component of $\mathbb{R}^{n+1} \backslash C_{i}$. Since $B_{i} \cap D_{i}$ is open and connected in $D_{i}$, and $D_{i}$ is homeomorphic to $S^{n}$, it follows that $B_{i} \cap D_{i}$ is path connected and we can use the strong local homogeneity of $S^{n}$ to see that there is a homeomorphism $g_{i}: D_{i} \rightarrow D_{i}$, with $g_{i}(x)=y$, supported on $B_{i} \cap D_{i}$. By Theorem 2.3 we can extend $g_{i}$ to a homeomorphism $\overline{g_{i}}$ of $C_{i}$. If we now define $h_{i}: M_{n}^{n+1} \rightarrow M_{n}^{n+1}$ by

$$
h_{i}(x)= \begin{cases}\overline{g_{i}}(x) & \text { if } x \in C_{i}, \\ x & \text { otherwise }\end{cases}
$$

then $h_{i}$ is as required. 
We want to derive a similar result for the interior points of $M_{n}^{n+1}$ with $n \in \mathbb{N} \backslash\{3\}$. For this we use the following lemma. Recall that $\partial\left(I^{n+1}\right)$ is the boundary of the unbounded component of $\mathbb{R}^{n+1} \backslash M_{n}^{n+1}$.

Lemma 2.7. Let $n \in \mathbb{N} \backslash\{3\}$ and let $x$ and $y$ be interior points of $M_{n}^{n+1}$. Then there is a homeomorphism $h: M_{n}^{n+1} \rightarrow M_{n}^{n+1}$ with $h(x)=y$ and $h \uparrow \partial\left(I^{n+1}\right)=e_{\partial\left(I^{n+1}\right)}$.

Proof. If $x=y$ we can take $h=e_{M_{n}^{n+1}}$, so suppose that $x \neq y$. Clearly, we can find quotient mappings $q_{x}, q_{y}: \mathbb{R}^{n+1} \rightarrow \mathbb{R}^{n+1}$ with $q_{x}^{-1}(\{x\})=I^{n+1}$ and $q_{y}^{-1}(\{y\})=I^{n+1}$ such that $q_{x}: \mathbb{R}^{n+1} \backslash I^{n+1} \rightarrow \mathbb{R}^{n+1} \backslash\{x\}$ and $q_{y}: \mathbb{R}^{n+1} \backslash$ $I^{n+1} \rightarrow \mathbb{R}^{n+1} \backslash\{y\}$ are homeomorphisms. Then $q_{x}^{-1}\left(M_{n}^{n+1}\right) \backslash \operatorname{Int} I^{n+1}$ and $q_{y}^{-1}\left(M_{n}^{n+1}\right) \backslash$ Int $I^{n+1}$ are Sierpiński carpets and we denote them by $S_{x}$, respectively $S_{y}$.

Let $B_{x}$, respectively $B_{y}$, be the boundary of the unbounded component of $\mathbb{R}^{n+1} \backslash S_{x}$, respectively $\mathbb{R}^{n+1} \backslash S_{y}$. So $B_{x}=q_{x}^{-1}\left(\partial I^{n+1}\right)$ and $B_{y}=q_{y}^{-1}\left(\partial I^{n+1}\right)$. Note that $g=\left(q_{y}^{-1} \circ q_{x}\right)\left\lceil B_{x}\right.$ is a homeomorphism from $B_{x}$ to $B_{y}$ such that $q_{y} \circ g=q_{x}\left\lceil B_{x}\right.$. It follows from Remark 2.4 that we can extend $g$ to a homeomorphism $\bar{g}: S_{x} \rightarrow S_{y}$ such that $\bar{g}\left(\partial I^{n+1}\right)=\partial I^{n+1}$.

Now define the function $h: M_{n}^{n+1} \rightarrow M_{n}^{n+1}$ by

$$
h(z)= \begin{cases}y & \text { if } z=x, \\ \left(q_{y} \circ \bar{g} \circ q_{x}^{-1}\right)(z) & \text { if } z \neq x .\end{cases}
$$

It is easy to see that $h$ is a bijection such that $h \circ q_{x}=q_{y} \circ \bar{g}$. Since $q_{x}$ is a quotient mapping and $q_{y} \circ \bar{g}$ is continuous, it follows that $h$ is continuous. By compactness of $M_{n}^{n+1}$ we see that $h$ is a homeomorphism.

Take $z \in \partial\left(I^{n+1}\right)$. Then $q_{x}^{-1}(z) \in B_{x}$ and since $\bar{g}$ is an extension of $g$ we see that

$$
h(z)=\left(q_{y} \circ \bar{g}\right)\left(q_{x}^{-1}(z)\right)=\left(q_{y} \circ g\right)\left(q_{x}^{-1}(z)\right)=q_{x}\left(q_{x}^{-1}(z)\right)=z .
$$

This shows that $h\left\lceil\partial\left(I^{n+1}\right)=e_{\partial\left(I^{n+1}\right)}\right.$, so $h$ is as required.

Lemma 2.8. Let $n \in \mathbb{N} \backslash\{3\}$ and suppose that $x$ is an interior point of $M_{n}^{n+1}$. Then there is a local basis $\mathcal{B}_{x}$ at $x$ such that for every $B \in \mathcal{B}_{x}$ and every interior point $y$ of $M_{n}^{n+1}$ in $B$ there is a homeomorphism $h$ of $M_{n}^{n+1}$ with $h(x)=y$ that is supported on $B$.

Proof. It follows from the construction of $M_{n}^{n+1}$ that $x$ has arbitrarily small open neighbourhoods $B$ in $M_{n}^{n+1}$ such that $\bar{B}$, the closure of $B$ in $M_{n}^{n+1}$ (or in $\mathbb{R}^{n+1}$ ), is homeomorphic to $M_{n}^{n+1}$ and the boundary $\partial B$ of $B$ in $M_{n}^{n+1}$ is the boundary of the unbounded component of $\mathbb{R}^{n+1} \backslash \bar{B}$. Let $\mathcal{B}_{x}$ be the collection of those neighbourhoods $B$ of $x$. Clearly, $\mathcal{B}_{x}$ is a local basis at $x$. If $y$ is an interior point of $M_{n}^{n+1}$ such that $y$ is an element of a $B \in \mathcal{B}_{x}$, then $y$ is an interior point of $\frac{n}{B}$. It follows from Lemma 2.7 that we can find a homeomorphism of $\bar{B}$ that maps $x$ to $y$ and is the identity 
on the boundary of $B$ in $M_{n}^{n+1}$. This homeomorphism can be extended to $M_{n}^{n+1}$ by taking the identity on $M_{n}^{n+1} \backslash \bar{B}$. This shows that the local basis $\mathcal{B}_{x}$ at $x$ is as required.

Lemma 2.9. Let $O$ be an open subset of $M_{n}^{n+1}$ for $n \in \mathbb{N} \backslash\{3\}$ and let $D_{1}$ and $D_{2}$ be countable subsets of $O$. Suppose that for $j \in\{1,2\}$ the interior points of $M_{n}^{n+1}$ contained in $D_{j}$ are dense in $O$, and $D_{j} \cap \partial U_{i}$ is dense in $\partial U_{i} \cap O$ for all $i$. Then there is a homeomorphism $h$ of $M_{n}^{n+1}$ that is supported on $O$ and satisfies $h\left(D_{1}\right)=D_{2}$.

Proof. This proof uses a well known back-and-forth construction; see for instance [1] or [8, Theorem 1.6.9]. Write $D_{1}=D_{1}^{\mathrm{i}} \cup D_{1}^{\mathrm{b}}$, where $D_{1}^{\mathrm{i}}$ is the set of all points of $D_{1}$ that are interior points of $M_{n}^{n+1}$, and $D_{1}^{\mathrm{b}}$ is the set of all points of $D_{1}$ that are boundary points of $M_{n}^{n+1}$. Similarly, write $D_{2}=D_{2}^{\mathrm{i}} \cup D_{2}^{\mathrm{b}}$. Let $\left\{a_{1}, a_{2}, \ldots\right\}$ and $\left\{\tilde{a}_{1}, \tilde{a}_{2}, \ldots\right\}$ be enumerations of $D_{1}^{\mathrm{i}}$, respectively $D_{1}^{\mathrm{b}}$, and let $\left\{b_{1}, b_{2}, \ldots\right\}$ and $\left\{\tilde{b}_{1}, \tilde{b}_{2}, \ldots\right\}$ be enumerations of $D_{2}^{\mathrm{i}}$, respectively $D_{2}^{\mathrm{b}}$. Using the Inductive Convergence Criterion [8, 1.6.2] we construct a sequence $\left(h_{m}\right)_{m \in \mathbb{N}}$ of homeomorphisms of $M_{n}^{n+1}$ such that $h=\lim _{m \rightarrow \infty} h_{m} \circ \cdots \circ h_{1}$ exists and is a homeomorphism, and the following conditions are satisfied:

(1) $h_{m}$ is supported on $O$ for all $m \in \mathbb{N}$;

(2) $h_{m} \circ \cdots \circ h_{1}\left(a_{j}\right)=h_{4 j-2} \circ \cdots \circ h_{1}\left(a_{j}\right) \in D_{2}^{\mathrm{i}}$ for all $j$ and $m \geq 4 j-2$;

(3) $\left(h_{m} \circ \cdots \circ h_{1}\right)^{-1}\left(b_{j}\right)=\left(h_{4 j-1} \circ \cdots \circ h_{1}\right)^{-1}\left(b_{j}\right) \in D_{1}^{\mathrm{i}}$ for all $j$ and $m \geq 4 j-1$

(4) $h_{m} \circ \cdots \circ h_{1}\left(\tilde{a}_{j}\right)=h_{4 j} \circ \cdots \circ h_{1}\left(\tilde{a}_{j}\right) \in D_{2}^{\mathrm{b}}$ for all $j$ and $m \geq 4 j$;

(5) $\left(h_{m} \circ \cdots \circ h_{1}\right)^{-1}\left(\tilde{b}_{j}\right)=\left(h_{4 j+1} \circ \cdots \circ h_{1}\right)^{-1}\left(\tilde{b}_{j}\right) \in D_{1}^{\mathrm{b}}$ for all $j$ and $m \geq 4 j+1$.

These conditions ensure that $h \in \mathcal{H}_{O}\left(M_{n}^{n+1}\right), h\left(D_{1}^{\mathrm{i}}\right)=D_{2}^{\mathrm{i}}$ and $h\left(D_{1}^{\mathrm{b}}\right)=D_{2}^{\mathrm{b}}$. Put $h_{1}=e_{M_{n}^{n+1}}$ and assume that $h_{1}, \ldots, h_{4 j-3}$ are defined for certain $j \in \mathbb{N}$.

If $h_{4 j-3} \circ \cdots \circ h_{1}\left(a_{j}\right) \in D_{2}^{\mathrm{i}}$, take $h_{4 j-2}=e_{M_{n}^{n+1}}$. Otherwise, we use Lemma 2.8 to find a small neighbourhood $V_{4 j-2} \subset O$ of $h_{4 j-3} \circ \cdots \circ h_{1}\left(a_{j}\right)$ which is disjoint from the finite set

$$
\left\{b_{1}, \ldots, b_{j-1}, \tilde{b}_{1}, \ldots, \tilde{b}_{j-1}\right\} \cup h_{4 j-3} \circ \cdots \circ h_{1}\left(\left\{a_{1}, \ldots, a_{j-1}, \tilde{a}_{1}, \ldots \tilde{a}_{j-1}\right\}\right)
$$

and moreover has the property that we can map $h_{4 j-3} \circ \cdots \circ h_{1}\left(a_{j}\right)$ to any other interior point of $M_{n}^{n+1}$ in $V_{4 j-2}$ by a homeomorphism supported on $V_{4 j-2}$. Since $D_{2}^{\mathrm{i}}$ is dense in $O$ we have $D_{2}^{\mathrm{i}} \cap V_{4 j-2} \neq \emptyset$. This means that we can find a homeomorphism $f_{4 j-2}$ of $M_{n}^{n+1}$ supported on $V_{4 j-2}$ such that

$$
f_{4 j-2} \circ h_{4 j-3} \circ \cdots \circ h_{1}\left(a_{j}\right) \in D_{2}^{\mathrm{i}} .
$$

We put $h_{4 j-2}=f_{4 j-2}$.

If $\left(h_{4 j-2} \circ \cdots \circ h_{1}\right)^{-1}\left(b_{j}\right) \in D_{1}^{\mathrm{i}}$, we take $h_{4 j-1}=e_{M_{n}^{n+1}}$. Otherwise, we use Lemma 2.8 again to find a small neighbourhood $V_{4 j-1} \subset O$ of $b_{j}$ that is 
disjoint from the finite set

$$
\left\{b_{1}, \ldots, b_{j-1}, \tilde{b}_{1}, \ldots, \tilde{b}_{j-1}\right\} \cup h_{4 j-2} \circ \cdots \circ h_{1}\left(\left\{a_{1}, \ldots, a_{j}, \tilde{a}_{1}, \ldots \tilde{a}_{j-1}\right\}\right)
$$

and has the property that we can map $b_{j}$ to any other interior point of $M_{n}^{n+1}$ in $V_{4 j-1}$ by a homeomorphism supported on $V_{4 j-1}$. Since $\left(h_{4 j-2} \circ \cdots \circ h_{1}\right)\left(D_{1}^{\mathrm{i}}\right)$ is dense in $O$, by 22 we know that $\left(h_{4 j-2} \circ \cdots \circ h_{1}\right)\left(D_{1}^{\mathrm{i}}\right) \cap V_{4 j-1} \neq \emptyset$. This means that there is a homeomorphism $f_{4 j-1}$ of $M_{n}^{n+1}$ supported on $V_{4 j-1}$ such that

$$
f_{4 j-1}^{-1}\left(b_{j}\right) \in\left(h_{4 j-2} \circ \cdots \circ h_{1}\right)\left(D_{1}^{\mathrm{i}}\right) .
$$

We put $h_{4 j-1}=f_{4 j-1}$.

Using the same argument as above, but now applying Lemma 2.6 instead of Lemma 2.8, we find neighbourhoods $V_{4 j}, V_{4 j+1} \subset O$ of $\left(h_{4 j-1} \circ \cdots \circ h_{1}\right)\left(\tilde{a}_{j}\right)$, respectively $b_{j}$, and homeomorphisms $h_{4 j}, h_{4 j+1}$ in $\mathcal{H}_{V_{4 j}}\left(M_{n}^{n+1}\right)$, respectively $\mathcal{H}_{V_{4 j+1}}\left(M_{n}^{n+1}\right)$, such that

$$
h_{4 j} \circ h_{4 j-1} \circ \cdots \circ h_{1}\left(\tilde{a}_{j}\right) \in D_{2}^{\mathrm{b}}, \quad h_{4 j+1}^{-1}\left(\tilde{b}_{j}\right) \in h_{4 j} \circ \cdots \circ h_{1}\left(D_{1}^{\mathrm{b}}\right) .
$$

If the neighbourhoods $V_{4 j-2}, V_{4 j-1}, V_{4 j}, V_{4 j+1}$ are chosen small enough, then the conditions of the Inductive Convergence Criterion are satisfied.

REMARK 2.10. It follows immediately from this lemma that if $D_{1} \cap \partial U_{i}$ and $D_{2} \cap \partial U_{i}$ are dense in $\partial U_{i} \cap O$ for every $i$ with $\partial U_{i} \cap O \neq \emptyset$, and $D_{1}$ and $D_{2}$ do not contain any interior points of $M_{n}^{n+1}$, there is a homeomorphism $h$ of $M_{n}^{n+1}$ supported on $O$ that maps $D_{1}$ onto $D_{2}$. Similarly, if $D_{1}$ and $D_{2}$ both entirely consist of interior points of $M_{n}^{n+1}$, there also exists such a homeomorphism.

Now let $p \geq 1$ and consider the Banach space $\ell^{p}$ of all sequences $z=$ $\left(z_{0}, z_{1}, \ldots\right) \in \mathbb{R}^{\omega}$ such that $\sum_{n=0}^{\infty}\left|z_{n}\right|^{p}<\infty$. The topology on $\ell^{p}$ is generated by the $p$-norm $\|z\|_{p}=\left(\sum_{n=0}^{\infty}\left|z_{n}\right|^{p}\right)^{1 / p}$. It is well known that $\|\cdot\|_{p}$ is a Kadec norm with respect to the coordinate projections, that is, the norm topology is the weakest topology that makes all the coordinate projections $z \mapsto z_{n}$ and the norm function continuous. This fact can also be formulated as follows: the norm topology on $\ell^{p}$ is generated by the product topology (inherited from $\mathbb{R}^{\omega}$ ) together with the sets $\left\{z \in \ell^{p}:\|z\|_{p}<t\right\}$ for $t>0$. We extend the $p$-norm over $\hat{\mathbb{R}}^{\omega}$ by putting $\|z\|_{p}=\infty$ for each $z \in \hat{\mathbb{R}}^{\omega} \backslash \ell^{p}$.

Definition 2.11. Let $X$ be a space. A function $f: X \rightarrow \hat{\mathbb{R}}$ is called lower semicontinuous (abbreviated $L S C$ ) if $f^{-1}((t, \infty])$ is open in $X$ for every $t \in \mathbb{R}$.

Note that the norm as a function from $\hat{\mathbb{R}}^{\omega}$ to $[0, \infty]$ is LSC but not continuous because the norm topology on $\ell^{p}$ is much stronger than the topology inherited from $\mathbb{R}^{\omega}$. It is easily checked that $f: X \rightarrow \hat{\mathbb{R}}$ is LSC if 
and only if $f\left(\lim _{n \rightarrow \infty} x_{n}\right) \leq \liminf _{n \rightarrow \infty} f\left(x_{n}\right)$ for every convergent sequence $\left(x_{n}\right)_{n \in \omega}$ in $X$.

We define Erdős space

$$
\mathfrak{E}=\left\{x \in \ell^{2}: x_{n} \in \mathbb{Q} \text { for all } n \in \omega\right\} .
$$

Let $\mathcal{T}$ be the zero-dimensional topology that $\mathfrak{E}$ inherits from $\mathbb{Q}^{\omega}$. We noted that $\mathcal{T}$ is weaker than the norm topology, so clopen sets separate points, that is, $\mathfrak{E}$ is totally disconnected. By the remark above, the graph of the norm function, when seen as a function from $(\mathfrak{E}, \mathcal{T})$ to $\mathbb{R}^{+}$, is homeomorphic to $\mathfrak{E}$. This means that we can informally think of $\mathfrak{E}$ as a "zerodimensional space with some LSC function declared continuous".

We point out the following connection between the two topologies on $\mathfrak{E}$. Because the norm is LSC on $\mathbb{R}^{\omega}$, every closed $\varepsilon$-ball in $\mathfrak{E}$ is also closed in the zero-dimensional space $\mathbb{Q}^{\omega}$. This means that every point in $\mathfrak{E}$ has arbitrarily small neighbourhoods which are intersections of clopen sets.

Definition 2.12. A subset $A$ of a space $X$ is called a $C$-set in $X$ if $A$ can be written as an intersection of clopen subsets of $X$. A space is called $a l$ most zero-dimensional if every point of the space has a neighbourhood basis consisting of $C$-sets. If $Z$ is a set that contains $X$ then we say that a (separable metric) topology $\mathcal{T}$ on $Z$ witnesses the almost zero-dimensionality of $X$ if $\operatorname{dim}(Z, \mathcal{T}) \leq 0, O \cap X$ is open in $X$ for each $O \in \mathcal{T}$, and every point of $X$ has a neighbourhood basis in $X$ consisting of sets that are closed in $(Z, \mathcal{T})$. We will also say that the space $(Z, \mathcal{T})$ is a witness to the almost zero-dimensionality of $X$.

Thus $\mathfrak{E}$ is almost zero-dimensional. The space $\mathbb{Q}^{\omega}$ is a witness to the almost zero-dimensionality of Erdős space. More generally, if $\varphi: Z \rightarrow \mathbb{R}$ is an LSC function with a zero-dimensional domain then it follows easily that $Z$ is a witness to the almost zero-dimensionality of the graph of $\varphi$. Clearly, a space $X$ is almost zero-dimensional if and only if there is a topology on $X$ witnessing this fact. Oversteegen and Tymchatyn [10] proved that every almost zero-dimensional space is at most one-dimensional.

Definition 2.13. Let $X$ be a space and let $\mathcal{A}$ be a collection of subsets of $X$. The space $X$ is called $\mathcal{A}$-cohesive if every point of $X$ has a neighbourhood that does not contain nonempty clopen subsets of any element of $\mathcal{A}$. If a space $X$ is $\{X\}$-cohesive then we simply call $X$ cohesive.

Again, let $p \geq 1$. As a generalization of the construction of $\mathfrak{E}$, consider a fixed sequence $E_{0}, E_{1}, E_{2}, \ldots$ of subsets of $\mathbb{R}$ and let

$$
\mathcal{E}=\left\{z \in \ell^{p}: z_{n} \in E_{n} \text { for every } n \in \omega\right\} .
$$

The following two results were proved in Dijkstra [3. 
Theorem 2.14. Assume that $\mathcal{E}$ is not empty and that every $E_{n}$ is zerodimensional. The following statements are equivalent:

(1) there exists an $x \in \prod_{n=0}^{\infty} E_{n}$ with $\|x\|_{p}=\infty$ and $\lim _{n \rightarrow \infty} x_{n}=0$,

(2) every nonempty clopen subset of $\mathcal{E}$ is unbounded,

(3) $\mathcal{E}$ is cohesive,

(4) $\operatorname{dim} \mathcal{E}>0$.

Recall that if $A_{0}, A_{1}, \ldots$ is a sequence of subsets of a space $X$ then $\lim \sup _{n \rightarrow \infty} A_{n}=\bigcap_{n=0}^{\infty} \frac{\bigcup_{k=n}^{\infty} A_{k}}{\text {. }}$

COROLlary 2.15. If 0 is a cluster point of $\lim \sup _{n \rightarrow \infty} E_{n}$ then every nonempty clopen subset of $\mathcal{E}$ is unbounded (and hence $\operatorname{dim} \mathcal{E} \neq 0$ ).

We need some new notions. The following definitions are taken from Dijkstra and van Mill [5].

Definition 2.16. If $A$ is a nonempty set then $A^{<\omega}$ denotes the set of all finite strings of elements of $A$, including the null string $\lambda$. If $s=$ $a_{0} a_{1} \ldots a_{k-1} \in A^{<\omega}$ for some $k \in \omega$, then $|s|$ denotes its length $k$. In this context the set $A$ is called an alphabet. Let $A^{\omega}$ denote the set of all infinite strings $a_{0} a_{1} \ldots$ of elements of $A$. If $s \in A^{<\omega}$ and $\sigma \in A^{<\omega} \cup A^{\omega}$ then we put $s \prec \sigma$ if $s$ is an initial substring of $\sigma$, that is, there is a $\tau \in A^{<\omega} \cup A^{\omega}$ with $s^{\frown} \tau=\sigma$, where $\frown$ denotes concatenation of strings. If $\sigma=a_{0} a_{1} \ldots \in$ $A^{<\omega} \cup A^{\omega}$ and $k \in \omega$ with $k \leq|\sigma|$, then $\sigma\left\lceil k=a_{0} a_{1} \ldots a_{k-1}\right.$.

Definition 2.17. A tree $T$ on an alphabet $A$ is a subset of $A^{<\omega}$ that is closed under initial segments, that is, if $s \in T$ and $t \prec s$ then $t \in T$. An infinite branch of $T$ is an element $\sigma$ of $A^{\omega}$ such that $\sigma\lceil k \in T$ for every $k \in \omega$. The body of $T$, written as $[T]$, is the set of all infinite branches of $T$. If $s, t \in T$ are such that $s \prec t$ and $|t|=|s|+1$ then we say that $t$ is an immediate successor of $s$, and $\operatorname{succ}(s)$ denotes the set of immediate successors of $s$ in $T$.

Now we introduce the concept of an anchor.

Definition 2.18. Let $T$ be a tree and let $\left(X_{s}\right)_{s \in T}$ be a system of subsets of a space $X$ such that $X_{t} \subset X_{s}$ whenever $s \prec t$. A subset $A$ of $X$ is called an anchor for $\left(X_{s}\right)_{s \in T}$ in $X$ if for every $\sigma \in[T]$, the sequence $X_{\sigma\lceil 0}, X_{\sigma\lceil 1}, \ldots$ converges to a point in $X$ whenever $X_{\sigma \uparrow k} \cap A \neq \emptyset$ for all $k \in \omega$.

EXAMPLE 2.19. As noted before, $\mathbb{Q}^{\omega}$ is a witness to the almost zerodimensionality of $\mathfrak{E}$. Let $\mathcal{T}$ be the topology that $\mathfrak{E}$ inherits from $\mathbb{Q}^{\omega}$. Put $T=\mathbb{Q}^{<\omega}$, and for $s=q_{0} \ldots q_{k-1} \in T$ with $k \in \omega$, let $\mathbb{Q}_{s}^{\omega}$ be the closed subset of $\mathbb{Q}^{\omega}$ given by

$$
\mathbb{Q}_{s}^{\omega}=\left\{x \in \mathbb{Q}^{\omega}: x_{i}=q_{i} \text { for } 0 \leq i \leq k-1\right\} .
$$


Put $\mathfrak{E}_{s}=\mathbb{Q}_{s}^{\omega} \cap \mathfrak{E}$ for $s \in T$ and let $B$ be a bounded subset of $\mathfrak{E}$. We show that $B$ is an anchor for $\left(\mathfrak{E}_{s}\right)_{s \in T}$ in $(\mathfrak{E}, \mathcal{T})$. Let $\sigma=q_{0} q_{1} \ldots \in[T]$ be such that $\mathfrak{E}_{\sigma \mid k} \cap B \neq \emptyset$ for all $k \in \omega$. It is clear that $\mathfrak{E}_{\sigma\lceil k}$ converges to the point $\sigma \in \mathbb{Q}^{\omega}$ in the product topology of $\mathbb{Q}^{\omega}$, where we identify the string $q_{0} q_{1} \ldots$ with the sequence $\left(q_{0}, q_{1}, \ldots\right)$. It suffices to show that $\sigma \in \mathfrak{E}$. Since $B$ is bounded there is an $M \in \mathbb{N}$ such that $B \subset\left\{x \in \mathbb{Q}^{\omega}:\|x\| \leq M\right\}$, and because $\mathfrak{E}_{\sigma \mid k} \cap B \neq \emptyset$ for all $k \in \omega$ this means that $\left\|\left(q_{0}, q_{1}, \ldots, q_{k-1}, 0,0, \ldots\right)\right\| \leq M$ for all $k \geq 0$. We have

$$
\|\sigma\|=\lim _{k \rightarrow \infty}\left\|\left(q_{0}, q_{1}, \ldots, q_{k-1}, 0,0, \ldots\right)\right\| \leq M,
$$

so $\sigma \in \mathfrak{E}$.

Dijkstra and van Mill [5, §8] introduced the following class $E^{\prime}$ of spaces.

DeFinition 2.20. $\mathrm{E}^{\prime}$ is the class of all nonempty spaces $E$ such that there exists an $F_{\sigma \delta}$-topology $\mathcal{T}$ on $E$ that witnesses the almost zero-dimensionality of $E$ and there exist a nonempty tree $T$ over a countable set and subspaces $E_{s}$ of $E$ that are closed with respect to $\mathcal{T}$ for each $s \in T \backslash\{\lambda\}$ such that

$\left(1^{\prime}\right) E_{\lambda}$ is dense in $E$ and $E_{s}=\bigcup\left\{E_{t}: t \in \operatorname{succ}(s)\right\}$ whenever $s \in T$,

$\left(2^{\prime}\right)$ each $x \in E$ has a neighbourhood $U$ that is an anchor for $\left(E_{s}\right)_{s \in T}$ in $(E, \mathcal{T})$

$\left(3^{\prime}\right)$ for each $s \in T \backslash \lambda$ and $t \in \operatorname{succ}(s), E_{t}$ is nowhere dense in $E_{s}$,

$\left(4^{\prime}\right) E$ is $\left\{E_{s}: s \in T\right\}$-cohesive,

$\left(5^{\prime}\right) E$ can be written as a countable union of nowhere dense subsets that are closed with respect to $\mathcal{T}$.

In [5, Theorem 8.13] Dijkstra and van Mill prove

Theorem 2.21. A space $E$ is homeomorphic to $\mathfrak{E}$ if and only if $E \in \mathrm{E}^{\prime}$.

As an illustration we show that $\mathfrak{E}$ satisfies the conditions of Definition 2.20 . Let $\mathcal{T}$ be the product topology that $\mathfrak{E}$ inherits from $\mathbb{Q}^{\omega}$, put $T=\mathbb{Q}^{<\omega}$ and let $\mathfrak{E}_{s}$ for $s \in T$ be as defined in Example 2.19. Since $\mathbb{Q}$ is a $\sigma$-compact space, it is easy to see that $\mathbb{Q}^{\omega}$ is an absolute $F_{\sigma \delta}$-space. Furthermore, $\mathfrak{E}$ is an $F_{\sigma}$ subset of $\mathbb{Q}^{\omega}$, which means that $\mathcal{T}$ is indeed an $F_{\sigma \delta}$-topology on $\mathfrak{E}$ that witnesses the almost zero-dimensionality of $\mathfrak{E}$. It is clear that $\mathfrak{E}_{s}$ is closed in $(\mathfrak{E}, \mathcal{T})$ for all $s \in T$ and conditions $\left(1^{\prime}\right),\left(3^{\prime}\right)$ and $\left(5^{\prime}\right)$ are easily seen to be satisfied. For $\left(2^{\prime}\right)$ and $\left(4^{\prime}\right)$ note that it follows from Example 2.19 and Corollary 2.15 that every bounded neighbourhood of a point $x$ in $\mathfrak{E}$ is an anchor for $\left(\mathfrak{E}_{s}\right)_{s \in T}$ in $(\mathfrak{E}, \mathcal{T})$ that contains no nonempty clopen subsets of any $\mathfrak{E}_{s}$.

3. Homeomorphism groups of a Sierpiński carpet. We prove the following theorem for $n$-dimensional Sierpiński carpets as an extension of the results in [5, Chapter 10]. 
Theorem 3.1. Let $n \in \mathbb{N} \backslash\{3\}$, let $\left\{U_{i}: i \in \mathbb{N}\right\}$ be the collection of components of $S^{n+1} \backslash M_{n}^{n+1}$, and let $D$ be a countable dense subset of $M_{n}^{n+1}$. If $O$ is a nonempty open subset of $M_{n}^{n+1}$ such that either $D \cap \partial U_{i}=\emptyset$ for every $i$ with $\partial U_{i} \subset O$, or $D \cap \partial U_{i}$ is dense in $\partial U_{i}$ for every $i$ with $\partial U_{i} \subset O$, then $\mathcal{H}_{U}\left(M_{n}^{n+1}, D\right)$ is homeomorphic to Erdös space for every open $U$ that contains $O$.

As noted before, $M_{n}^{n+1}$ is not homogeneous, which is why we need the conditions on $D$ here. If we choose for instance a set $D \subset M_{n}^{n+1}$ such that $\left|D \cap \partial U_{i}\right|=i$ for every $i$ then $\mathcal{H}\left(M_{n}^{n+1}, D\right)$ contains only the identity map.

Note that if $D \cap \partial U_{i}=\emptyset$ for all $\partial U_{i} \subset O$, there can still be $j \in \mathbb{N}$ with $D \cap \partial U_{j} \cap O \neq \emptyset$. Similarly, if $D \cap \partial U_{i}$ is dense in $\partial U_{i}$ for all $\partial U_{i} \subset O$, there can still be $j \in \mathbb{N}$ such that $D \cap \partial U_{j} \cap O$ is not dense in $\partial U_{j} \cap O$. The following claim shows that for the proof of Theorem 3.1 we can avoid these situations. Furthermore, it shows that if $D \cap \partial U_{i}$ is dense in $\partial U_{i}$ for all $\partial U_{i} \subset O$, we may assume that the set of interior points of $M_{n}^{n+1}$ contained in $D \cap O$ is either empty or dense in $O$. This observation will also be useful in the proof of Theorem 3.1 .

Claim 3.2. It suffices to prove Theorem 3.1 for the following three cases:

(i) $D \cap O$ consists entirely of interior points of $M_{n}^{n+1}$;

(ii) $D \cap \partial U_{i} \cap O$ is dense in $\partial U_{i} \cap O$ for every $i \in \mathbb{N}$ and the interior points of $M_{n}^{n+1}$ contained in $D \cap O$ are dense in $O$;

(iii) $D \cap \partial U_{i} \cap O$ is dense in $\partial U_{i} \cap O$ for every $i \in \mathbb{N}$ and $D \cap O$ contains no interior points of $M_{n}^{n+1}$.

Proof. Suppose that we are in the situation of Theorem 3.1. Let $D_{\mathrm{i}}$ be the set of all points of $D$ that are interior points of $M_{n}^{n+1}$. We define $O^{\prime} \subset O$ by

$$
O^{\prime}= \begin{cases}O \backslash \overline{D_{\mathrm{i}}} & \text { if } O \backslash \overline{D_{\mathrm{i}}} \neq \emptyset \\ O & \text { otherwise. }\end{cases}
$$

Clearly, $O^{\prime}$ is a nonempty open subset of $M_{n}^{n+1}$ such that either $D_{\mathrm{i}} \cap O^{\prime}=\emptyset$ or $D_{\mathrm{i}} \cap O^{\prime}$ is dense in $O^{\prime}$. Next we define $O^{\prime \prime} \subset O^{\prime}$ by

$$
O^{\prime \prime}=O^{\prime} \backslash \bigcup\left\{\partial U_{i}: \partial U_{i} \backslash O^{\prime} \neq \emptyset\right\} .
$$

Since the interior points of $M_{n}^{n+1}$ are dense in $M_{n}^{n+1}$ and the collection $\left\{U_{i}: i \in \mathbb{N}\right\}$ forms a null sequence, it follows that $O^{\prime \prime}$ is a nonempty open subset of $M_{n}^{n+1}$. Furthermore, if $\partial U_{i} \cap O^{\prime \prime} \neq \emptyset$ then $\partial U_{i} \subset O^{\prime \prime} \subset O$. It is clear that $O^{\prime \prime}$ satisfies one of the conditions (i), (ii) or (iii), and if we prove the theorem for $O^{\prime \prime}$ then we will have also proved it for $O$.

We introduce some notation. 
Definition 3.3. We define subspaces $E_{2}$ and $E_{4}$ of $\ell^{1}$ as follows:

$$
\begin{aligned}
& E_{2}=\left\{z \in \ell^{1}: 2^{i} z_{i} \in \omega \text { for all } i \in \omega\right\}, \\
& E_{4}=\left\{z \in \ell^{1}: 4^{i} z_{i} \in \omega \text { for all } i \in \omega\right\} .
\end{aligned}
$$

We write $Z_{2}$ for the space consisting of the set $E_{2}$ equipped with the zero-dimensional topology inherited from the product space $\mathbb{R}^{\omega}$, that is, the topology generated by the coordinate projections. Similarly, we write $Z_{4}$ for the set $E_{4}$ equipped with the zero-dimensional topology inherited from $\mathbb{R}^{\omega}$. For $i \in \omega$ we let $\xi_{i}: E_{4} \rightarrow E_{4}$ denote the projection given by $\xi_{i}(z)=\left(z_{0}, z_{1}, \ldots, z_{i}, 0,0, \ldots\right)$.

We will use the following proposition in the proof of Theorem 3.1 .

Proposition 3.4. Let $n \in \mathbb{N} \backslash\{3\}$ and let $O \subset M_{n}^{n+1}$ be open and nonempty. Then there exists a closed imbedding $G: E_{4} \ni z \mapsto G_{z}$ $\in \mathcal{H}_{O}\left(M_{n}^{n+1}\right)$, a copy $\hat{\mathbb{R}}_{\mathrm{c}}$ of $\hat{\mathbb{R}}$ in $O$ and a sequence $p_{1}, p_{2}, \ldots \in O \backslash \hat{\mathbb{R}}_{\mathrm{c}}$ such that

(a) $\lim _{i \rightarrow \infty} p_{i}=0_{\mathrm{c}} \in \mathbb{R}_{\mathrm{c}}$, where $\mathbb{R}_{\mathrm{c}}=\hat{\mathbb{R}}_{\mathrm{c}} \backslash\left\{ \pm \infty_{\mathrm{c}}\right\}$,

(b) for each $r \in \hat{\mathbb{R}}_{\mathrm{c}}$ and $z \in E_{4}$ we have $G_{z}(r)=r+\|z\| \in \hat{\mathbb{R}}_{\mathrm{c}}$,

(c) for each $x \in M_{n}^{n+1} \backslash \mathbb{R}_{\mathrm{c}}$ there is an $i \in \omega$ such that $G_{z}(x)=G_{\xi_{i}(z)}(x)$ for every $z \in E_{4}$,

(d) $\beta \circ G: Z_{4} \rightarrow \beta\left(\mathcal{H}\left(M_{n}^{n+1}\right)\right)$ is a closed imbedding, where $A=$ $\left\{\infty_{\mathrm{c}}, p_{1}, p_{2}, \ldots\right\}$ and $\beta: \mathcal{H}\left(M_{n}^{n+1}\right) \rightarrow\left(M_{n}^{n+1}\right)^{A}$ is given by $\beta(h)=$ $h \uparrow A$ (the restriction of $h$ to $A$ is an element of the infinite product space $\left.\left(M_{n}^{n+1}\right)^{A}\right)$.

The sets $\mathbb{R}_{\mathrm{c}}$ and $A$ can be chosen such that either both consist of interior points of $M_{n}^{n+1}$ or both consist of boundary points of $M_{n}^{n+1}$. Moreover, for $n=1$ the sets $\mathbb{R}_{\mathrm{c}}$ and $A$ can be chosen such that $\mathbb{R}_{\mathrm{c}}$ consists of interior points of $M_{1}^{2}$ and $A$ consists of boundary points of $M_{1}^{2}$.

Proof. Dijkstra [4, Remark 3] showed that there exists a closed imbed$\operatorname{ding} \bar{H}$ of $E_{2}$ in $\mathcal{H}(\bar{B})$, where $\bar{B}$ is a topological copy of $M_{n}^{n+1}$ that contains a copy $\hat{\mathbb{R}}_{\mathrm{c}}$ of $\hat{\mathbb{R}}$ and a sequence $p_{1}, p_{2}, \ldots \in \bar{B} \backslash \hat{\mathbb{R}}_{\mathrm{c}}$ such that properties (a)-(d) are satisfied. Note that we can imbed $E_{4}$ in $E_{2}$ by the map $g: E_{4} \rightarrow E_{2}$ given by

$$
g\left(z_{0}, z_{1}, \ldots\right)=\left(z_{0}, 0, z_{1}, 0, z_{2}, 0, \ldots\right) .
$$

Now $g$ is even an isometry such that $g\left(E_{4}\right)$ is closed with respect to the weak and (therefore also) strong topology on $E_{2}$. This means that we may assume that $\bar{H}$ is a closed imbedding of $E_{4}$ in $\mathcal{H}(\bar{B})$ with properties (a)-(d). We prove the proposition for $n=1$ and $n \geq 2$ separately.

CASE I: $n \in \mathbb{N} \backslash\{1,3\}$. This is the easy case because in the construction of Dijkstra [4, $\S 5$ ] the points of $\hat{\mathbb{R}}_{\mathrm{c}} \cup\left\{p_{1}, p_{2}, \ldots\right\}$ all lie in the same 
boundary $\partial U$ of some component $U$ of the complement of $\bar{B}$. From 4 , Remark 4] it follows immediately that there is an imbedding $G$ as described in the proposition and such that $\mathbb{R}_{\mathrm{c}}$ and $A$ both consist of boundary points of $M_{n}^{n+1}$.

To show that there is also a suitable imbedding $G$ such that both $\mathbb{R}_{\mathrm{c}}$ and $A$ consist of interior points of $M_{n}^{n+1}$, we consider two disjoint copies $B_{1}, B_{2}$ of $\bar{B}$ in $S^{n+1}$. Let $\partial U_{1}$, respectively $\partial U_{2}$, be the boundary of the component of $S^{n+1} \backslash B_{1}$, respectively $S^{n+1} \backslash B_{2}$, that contains the set $\hat{\mathbb{R}}_{\mathrm{c}} \cup\left\{p_{1}, p_{2}, \ldots\right\}$ in $B_{1}$, respectively $B_{2}$. Then, using Theorem 2.3, we make a new Sierpiński carpet $B$ from $B_{1}$ and $B_{2}$ by identifying the points of $\partial U_{1}$ with the corresponding points on $\partial U_{2}$. This means that the set $\hat{\mathbb{R}}_{\mathrm{c}} \cup\left\{p_{1}, p_{2}, \ldots\right\} \subset B_{1}$ now only contains interior points of $B$. Dijkstra's imbeddings of $E_{4}$ in $\mathcal{H}\left(B_{1}\right)$ and $\mathcal{H}\left(B_{2}\right)$ naturally give rise to an imbedding $G$ of $E_{4}$ in $\mathcal{H}(B)$ that satisfies the requirements of the proposition and is such that $\mathbb{R}_{\mathrm{c}}$ and $A$ both consist of interior points of $B$. Applying [4, Remark 4] we see that there exists an imbedding $G$ as in the proposition with $\mathbb{R}_{\mathrm{c}}$ and $A$ both consisting of interior points of $M_{n}^{n+1}$.

CASE II: $n=1$. In this case the set $\hat{\mathbb{R}}_{\mathrm{c}}$ consists of boundary points of $\bar{B}$ and the sequence $p_{1}, p_{2}, \ldots$ consists, with the exception of one point, of interior points of $\bar{B}$; see [4, §5]. We note that all points of $\hat{\mathbb{R}}_{\mathrm{c}} \cup\left\{p_{1}, p_{2}, \ldots\right\}$ that are boundary points of $\bar{B}$ lie in the boundary of the same component of $S^{2} \backslash \bar{B}$. This means that we can use the same argument as in the case $n \in \mathbb{N} \backslash\{1,3\}$ to show that we can find an imbedding $G$ as required and such that $\mathbb{R}_{\mathrm{c}}$ and $A$ both consist of interior points of $M_{1}^{2}$.

Now we observe that it follows from the definition of $g$ and the construction of Dijkstra that all the points $p_{i}$ might as well be chosen as boundary points of $\bar{B}$. By [4, Remark 4] we can then find the desired imbedding $G$ with $\mathbb{R}_{\mathrm{c}}$ and $A$ both consisting of boundary points of $M_{1}^{2}$.

Consider now two disjoint copies $B_{1}, B_{2}$ of $\bar{B}$ in $S^{2}$ and assume that all the points $p_{i}$ in $B_{1}$ are boundary points. Let $\partial U_{1}$, respectively $\partial U_{2}$, be the boundary of the component of $S^{2} \backslash B_{1}$, respectively $S^{2} \backslash B_{2}$, that contains $\hat{\mathbb{R}}_{\mathrm{c}}$. The set $\hat{\mathbb{R}}_{\mathrm{c}}$ in $B_{1}$ is an arc in the simple closed curve $\partial U_{1}$, and similarly the set $\hat{\mathbb{R}}_{\mathrm{c}}$ in $B_{2}$ is an arc in $\partial U_{2}$. This means that, using Theorem 2.3, we can form a new Sierpiński carpet $B$ from $B_{1}$ and $B_{2}$ by simply identifying the points of the set $\hat{\mathbb{R}}_{\mathrm{c}}$ in $B_{1}$ with the corresponding points of the set $\hat{\mathbb{R}}_{\mathrm{c}}$ in $B_{2}$. Then $\mathbb{R}_{\mathrm{c}} \subset B$ consists of interior points of $B$ and the points $\pm \infty_{\mathrm{c}}$ are boundary points of $B$. Dijkstra's imbeddings of $E_{4}$ in $\mathcal{H}\left(B_{1}\right)$ and $\mathcal{H}\left(B_{2}\right)$ naturally extend to an imbedding $G$ of $E_{4}$ in $\mathcal{H}(B)$ that satisfies properties (a)-(d) and is such that $\mathbb{R}_{\mathrm{c}}$ consists of interior points of $B$, and $A$ consists of boundary points of $B$. Applying [4, Remark 4] we see that there exists an imbedding $G$ as in the proposition with $\mathbb{R}_{\mathrm{C}}$ 
consisting of interior points of $M_{1}^{2}$ and $A$ consisting of boundary points of $M_{1}^{2}$.

We can now give the proof of Theorem 3.1 .

Proof of Theorem 3.1. Take an open subset $U$ of $M_{n}^{n+1}$ that contains $O$. Let $\rho$ be a metric on $M_{n}^{n+1}$ and let $\hat{\rho}$ be the induced metric on $\mathcal{H}\left(M_{n}^{n+1}\right)$ : $\hat{\rho}(f, g)=\max _{x \in M_{n}^{n+1}} \rho(f(x), g(x))$ for $f, g \in \mathcal{H}\left(M_{n}^{n+1}\right)$. Note that $\hat{\rho}$ is rightinvariant: $\hat{\rho}(f \circ h, g \circ h)=\hat{\rho}(f, g)$ for any $h \in \mathcal{H}\left(M_{n}^{n+1}\right)$. We prove the theorem by showing that $\mathcal{H}_{U}\left(M_{n}^{n+1}, D\right)$ satisfies the conditions of Definition 2.20. The result then follows from Theorem 2.21. Without loss of generality we may assume that $D \cap\left(M_{n}^{n+1} \backslash U\right)$ is dense in $M_{n}^{n+1} \backslash U$. Let $\mathcal{T}$ be the topology that $\mathcal{H}_{U}\left(M_{n}^{n+1}, D\right)$ inherits from the zero-dimensional product space $D^{D}$ via the injection $h \mapsto h\lceil D$. It follows from [5, Theorem 10.1] that $\mathcal{T}$ is an $F_{\sigma \delta}$-topology that witnesses the almost zero-dimensionality of $\mathcal{H}_{U}\left(M_{n}^{n+1}, D\right)$.

Consider the spaces $E_{4}$ and $Z_{4}$ and the projection map $\xi_{i}: E_{4} \rightarrow E_{4}$ for $i \in \omega$ as given in Definition 3.3. We let $P$ be the countable dense subset $\bigcup_{i=0}^{\infty} \xi_{i}\left(E_{4}\right)$ of $E_{4}$. Consider now the Cantor set

$$
C^{\prime}=\left\{z \in E_{4}: z_{i} \in\left\{0,4^{-i}\right\} \text { for } i \in \omega\right\},
$$

and note that since $\sum_{i=0}^{\infty} 4^{-i}<\infty$, the norm topology and the product topology coincide on $C^{\prime}$. Let $\delta: C^{\prime} \rightarrow \mathbb{R}^{+}$be the imbedding given by the rule $\delta(z)=\|z\|$. We define $C=\delta\left(C^{\prime}\right), \gamma=\delta^{-1} \uparrow C$, and $Q=\delta\left(C^{\prime} \cap P\right)$. Thus $C$ is a Cantor set with $Q$ as a countable dense subset and $\|\gamma(r)\|=r$ for each $r \in C$. We define subspaces $\mathcal{E}_{\mathrm{c}}$ and $\mathcal{E}$ of $\ell^{1}$ by

$$
\mathcal{E}_{\mathrm{c}}=\left\{z \in \ell^{1}: z_{i} \in C \text { for } i \in \omega\right\}, \quad \mathcal{E}=\left\{z \in \ell^{1}: z_{i} \in Q \text { for } i \in \omega\right\} .
$$

The subscript $\mathrm{c}$ refers to the fact that $\mathcal{E}_{\mathrm{c}}$ is a complete space. We let $Z_{\mathrm{c}}$ and $Z$ stand for $\mathcal{E}_{\text {c }}$ respectively $\mathcal{E}$ with the witness topologies that these spaces inherit from $\mathbb{R}^{\omega}$. Let $\nu: \omega \times \omega \rightarrow \omega$ be a bijection such that $\nu(i, j) \geq j$ for all $i, j \in \omega$. We define an imbedding $\zeta: \mathcal{E}_{\mathrm{c}} \rightarrow E_{4}$ by the rule $(\zeta(z))_{\nu(i, j)}=$ $\left(\gamma\left(z_{i}\right)\right)_{j}$ for $z \in \mathcal{E}_{\mathrm{c}}$ and $i, j \in \omega$. It is clear from the definition and the fact that the norm and product topology coincide on the compactum $C^{\prime}$ that $\zeta: Z_{\mathrm{c}} \rightarrow Z_{4}$ is a closed imbedding. Note that $\|\zeta(z)\|=\|z\|$ for each $z \in \mathcal{E}_{\mathrm{c}}$, which implies that $\zeta$ is also a closed imbedding with respect to the norm topologies (recall that the norm topology is generated by the product topology together with the norm function).

We select a null sequence of nonempty open sets $V_{0}, V_{1}, \ldots$ whose closures are disjoint subsets of $O$. Put $V=\bigcup_{k=0}^{\infty} V_{k}$. Using Proposition 3.4 we can find for every $k \in \omega$ a closed imbedding $G^{k}: E_{4} \rightarrow \mathcal{H}_{V_{k}}\left(M_{n}^{n+1}\right)$, a copy $\hat{\mathbb{R}}_{k}$ of $\hat{\mathbb{R}}$ in $V_{k}$ and a sequence $p_{1}^{k}, p_{2}^{k}, \ldots \in V_{k} \backslash \hat{\mathbb{R}}_{k}$ such that conditions (a)-(d) of Proposition 3.4 with $\hat{\mathbb{R}}_{\mathrm{c}}$ replaced by $\hat{\mathbb{R}}_{k}$ and $p_{i}$ replaced by $p_{i}^{k}$, 
are satisfied for $G^{k}$. If $x \in \hat{\mathbb{R}}$ we write $x_{k}$ for the representation of $x$ in $\hat{\mathbb{R}}_{k}$. Let $A_{k}=\left\{\infty_{k}, p_{1}^{k}, p_{2}^{k}, \ldots\right\}$ and let $\beta_{k}: \mathcal{H}\left(M_{n}^{n+1}\right) \rightarrow\left(M_{n}^{n+1}\right)^{A_{k}}$ be given by $\beta_{k}(h)=h\left\lceil A_{k}\right.$. Then condition (d) of Proposition 3.4 is satisfied for $G^{k}$ with the set $A_{k}$ and the map $\beta_{k}$.

We now define $H: \mathcal{E}_{\mathrm{c}} \rightarrow \mathcal{H}_{V}\left(M_{n}^{n+1}\right)$ by

$$
H_{z}(x)= \begin{cases}G_{\zeta(z)}^{0}(x) & \text { if } x \in V_{0}, \\ G_{\gamma\left(z_{k-1}\right)}^{k}(x) & \text { if } x \in V_{k} \text { for some } k \in \mathbb{N}, \\ x & \text { if } x \in M_{n}^{n+1} \backslash V,\end{cases}
$$

for $z \in \mathcal{E}_{\mathrm{c}}$. Since the $V_{k}$ 's form a null sequence it is clear that every $H_{z}$ is a homeomorphism of $M_{n}^{n+1}$ and that $H_{z}$ depends continuously on $z \in \mathcal{E}_{\mathrm{c}}$. Let $\Pi: \mathcal{H}_{V}\left(M_{n}^{n+1}\right) \rightarrow \mathcal{H}_{V_{0}}\left(M_{n}^{n+1}\right)$ be the continuous map defined by $\Pi(h)=$ $\left(h \nmid V_{0}\right) \cup e_{M_{n}^{n+1} \backslash V_{0}}$. Since $\zeta$ and $G^{0}$ are closed imbeddings and $\Pi \circ H=G^{0} \circ \zeta$, Lemma 2.1 implies that $H: \mathcal{E}_{\mathrm{c}} \rightarrow \mathcal{H}_{U}\left(M_{n}^{n+1}\right)$ is also a closed imbedding. Now we consider the three cases of Claim 3.2 separately.

CASE (i). In this case $D \cap O$ consists entirely of interior points of $M_{n}^{n+1}$. Choose a $k \in \omega$. By Proposition 3.4 we can choose the imbedding $G^{k}$ in (1) such that $A_{k}$ and $\mathbb{R}_{k}$ consist of interior points of $M_{n}^{n+1}$. Note that $\mathbb{R}_{k}$ is a nowhere dense subset of $V_{k}$. This means that we can find a countable dense subset $D_{k}$ of $V_{k}$, consisting of interior points of $M_{n}^{n+1}$, with $D_{k} \cap \mathbb{R}_{k}=\emptyset$ and $A_{k} \subset D_{k}$. Since $P$ is countable and $G_{z}^{k}\left(\mathbb{R}_{k}\right)=\mathbb{R}_{k}$ for all $z \in E_{4}$ (see property (b) of Proposition 3.4), we may assume that $G_{z}^{k}\left(D_{k}\right)=D_{k}$ for each $z \in P$. Let $\mathbb{Q}_{4}$ be the additive group $\left\{i 4^{j}: i, j \in \mathbb{Z}\right\}$ and note that $C \cap \mathbb{Q}_{4}=Q$. Let $\mathbb{Q}_{4}^{k}$ be the copy of $\mathbb{Q}_{4}$ that lies in $\mathbb{R}_{k}$, so $\mathbb{Q}_{4}^{k}$ consists of interior points of $M_{n}^{n+1}$. As observed in Remark 2.10, we may assume that the set $D$ has the properties

$$
D \cap V_{0}=D_{0}, \quad D \cap V_{k}=D_{k} \cup \mathbb{Q}_{4}^{k} \quad \text { for } k \in \mathbb{N} .
$$

We verify that

$$
\mathcal{E}=\left\{z \in \mathcal{E}_{\mathrm{c}}: H_{z}(D)=D\right\}
$$

and hence that $H\left\lceil\mathcal{E}\right.$ is a closed imbedding of $\mathcal{E}$ into $\mathcal{H}_{U}\left(M_{n}^{n+1}, D\right)$ for $n \in \mathbb{N}$. If $H_{z} \in \mathcal{H}_{U}\left(M_{n}^{n+1}, D\right)$ and $k \in \mathbb{N}$ then by property (b) of Proposition 3.4 we have $H_{z}\left(0_{k}\right)=\left\|\gamma\left(z_{k-1}\right)\right\|=z_{k-1} \in \mathbb{Q}_{4}$. Since $z \in \mathcal{E}_{\mathrm{c}}$ we also have $z_{k-1} \in C$ and hence $z_{k-1} \in Q$. Thus $z \in \mathcal{E}$. Conversely, let $z \in \mathcal{E}$. If $x \in V_{k} \backslash \mathbb{R}_{k}$ for some $k \in \omega$ then by property (c) of Proposition 3.4 there is a $z^{\prime} \in P$ such that $H_{z}(x)=G_{z^{\prime}}^{k}(x)$. Since $G_{z^{\prime}}^{k}\left(D_{k}\right)=D_{k}$ it follows that $x \in D_{k}=D \cap V_{k} \backslash \mathbb{R}_{k}$ if and only if $H_{z}(x) \in D_{k}$. Note that $H_{z}\left(\mathbb{R}_{0}\right)=\mathbb{R}_{0}$ and that this set is disjoint from $D$. Consider finally the case that $x \in \mathbb{R}_{k}$ for $k \in \mathbb{N}$. Then $z_{k-1} \in Q \subset \mathbb{Q}_{4}$ and $H_{z}(x)=G_{\gamma\left(z_{k-1}\right)}^{k}(x)=x+\left\|\gamma\left(z_{k-1}\right)\right\|=x+z_{k-1}$, which is in $\mathbb{Q}_{4}$ if and only if $x \in \mathbb{Q}_{4}$. 
Remember that $\mathcal{T}$ is the topology on $\mathcal{H}_{U}\left(M_{n}^{n+1}, D\right)$ inherited from $D^{D}$. Let $\mathcal{T}^{\prime}$ be the topology that $\mathcal{H}\left(M_{n}^{n+1}\right)$ inherits from $\left(M_{n}^{n+1}\right)^{D}$ and note that $\mathcal{T}^{\prime}$ restricts to $\mathcal{T}$ on $\mathcal{H}_{U}\left(M_{n}^{n+1}, D\right)$. We first verify that $H: Z_{\mathrm{c}} \rightarrow$ $\left(\mathcal{H}\left(M_{n}^{n+1}\right), \mathcal{T}^{\prime}\right)$ is continuous. Let $x \in D$. If $x \notin V$ or if $x \in V_{k}$ for some $k \in \mathbb{N}$, then $H_{z}(x)$ depends on at most a single coordinate of $z$, so continuity with respect to the product topology is obvious. Let $x \in V_{0}$ and thus $x \in D_{0} \subset V_{0} \backslash \mathbb{R}_{0}$. Then by property (c) of Proposition 3.4. $G_{z^{\prime}}^{0}(x)$ depends on only finitely many coordinates of $z^{\prime} \in E_{4}$ and hence $H_{z}(x)=G_{\zeta(z)}^{0}(x)$ also depends on only finitely many coordinates of $z \in Z_{\mathrm{c}}$. This shows that $H$ is continuous with respect to the product topologies. From property (d) of Proposition 3.4 we find that $\beta_{0} \circ H=\beta_{0} \circ G^{0} \circ \zeta$ is a closed imbedding of $Z_{\mathrm{c}}$ into $\beta_{0}\left(\mathcal{H}\left(M_{n}^{n+1}\right)\right)$. Since $A_{0} \subset D$, the mapping $\beta_{0}:\left(\mathcal{H}\left(M_{n}^{n+1}\right), \mathcal{T}^{\prime}\right) \rightarrow$ $\left(M_{n}^{n+1}\right)^{A_{0}}$ is continuous. Thus from Lemma 2.1 we conclude that $H: Z_{\mathrm{c}} \rightarrow$ $\left(\mathcal{H}\left(M_{n}^{n+1}\right), \mathcal{T}^{\prime}\right)$ is a closed imbedding. Since $Z=H^{-1}\left(\mathcal{H}_{U}\left(M_{n}^{n+1}, D\right)\right)$, also $H\left\lceil Z\right.$ is a closed imbedding of $Z$ in $\left(\mathcal{H}_{U}\left(M_{n}^{n+1}, D\right), \mathcal{T}\right)$.

Consider the point $0_{1} \in \mathbb{Q}_{4}^{1} \subset \mathbb{R}_{1}$. For every $a \in D$ we define $\Gamma_{a}=\{h \in$ $\left.\mathcal{H}_{U}\left(M_{n}^{n+1}, D\right): h\left(0_{1}\right)=a\right\}$. Note that every $\Gamma_{a}$ is closed with respect to $\mathcal{T}$ and that $\bigcup_{a \in D} \Gamma_{a}=\mathcal{H}_{U}\left(M_{n}^{n+1}, D\right)$. For $i \in \mathbb{N}$, let $z^{i}=\left(4^{-i}, 0,0, \ldots\right) \in \mathcal{E}$ and let $h \in \Gamma_{a}$. Since $\lim _{i \rightarrow \infty} z^{i}=\mathbf{0}$, where $\mathbf{0}$ denotes the zero vector in $\mathbb{R}^{\omega}$, it follows that $\lim _{i \rightarrow \infty} h \circ H_{\mathbf{0}}^{-1} \circ H_{z^{i}}=h$ in $\mathcal{H}_{U}\left(M_{n}^{n+1}, D\right)$. However, $h \circ H_{\mathbf{0}}^{-1} \circ H_{z^{i}} \notin \Gamma_{a}$. To see this, note that it follows from Proposition 3.4 . property (b), that $H_{0} \uparrow \hat{\mathbb{R}}_{1}=e_{\hat{\mathbb{R}}_{1}}$ and $H_{z^{i}}\left(0_{1}\right)=\left(4^{-i}\right)_{1}$. This implies that $h\left(H_{\mathbf{0}}^{-1}\left(H_{z^{i}}\left(0_{1}\right)\right)\right)=h\left(\left(4^{-i}\right)_{1}\right) \not h\left(0_{1}\right)=a$. Thus $\Gamma_{a}$ is nowhere dense in $\mathcal{H}_{U}\left(M_{n}^{n+1}, D\right)$ and condition $\left(5^{\prime}\right)$ of Definition 2.20 is satisfied.

We now make an observation which will be the key to satisfying conditions $\left(2^{\prime}\right)$ and $\left(4^{\prime}\right)$ of Definition 2.20 .

Claim 3.5. If $A$ is an unbounded subset of $\mathcal{E}$ then

$$
\operatorname{diam}_{\hat{\rho}}\left\{H_{z}: z \in A\right\} \geq \rho\left(-\infty_{0}, \infty_{0}\right) .
$$

Proof. Let $z \in A$ and let $n \in \mathbb{N}$ be arbitrary. Select a $z^{n} \in A$ such that $\left\|z^{n}\right\|>\|z\|+2 n$. It follows from (1), condition (b) of Proposition 3.4 and the fact that $\|\zeta(z)\|=\|z\|$ for all $z \in \mathcal{E}_{\mathrm{c}}$ that

$$
H_{z}\left((-\|z\|-n)_{0}\right)=G_{\zeta(z)}^{0}\left((-\|z\|-n)_{0}\right)=-n_{0} .
$$

Similarly, we see that

We conclude that

$$
H_{z^{n}}\left((-\|z\|-n)_{0}\right)=\left(\left\|z^{n}\right\|-\|z\|-n\right)_{0} .
$$

$$
\begin{aligned}
\operatorname{diam}_{\hat{\rho}}\left\{H_{z}: z \in A\right\} & \geq \limsup _{n \rightarrow \infty} \hat{\rho}\left(H_{z}, H_{z^{n}}\right) \\
& \geq \lim _{n \rightarrow \infty} \rho\left(-n_{0},\left(\left\|z^{n}\right\|-\|z\|-n\right)_{0}\right)=\rho\left(-\infty_{0}, \infty_{0}\right),
\end{aligned}
$$

proving Claim 3.5 . 
Let $T=Q^{<\omega}$ and define for $s=q_{1} \ldots q_{k} \in T$ with $k \in \omega$ the subspace $\mathcal{E}_{s}$ of $\mathcal{E}$ by

$$
\mathcal{E}_{s}=\left\{z \in \mathcal{E}: z_{i-1}=q_{i} \text { for } 1 \leq i \leq k\right\} .
$$

With the same arguments as given after Theorem 2.21 we see that the spaces $\mathcal{E}_{s}$ satisfy the conditions of Definition 2.20, every bounded subset of $\mathcal{E}$ is an anchor for $\left(\mathcal{E}_{s}\right)_{s \in T}$ in $Z$, and every nonempty clopen subset of any $\mathcal{E}_{s}$ is unbounded. Let $J=\left\{f_{q}: q \in Q\right\}$ be a countable dense subset of $\mathcal{H}_{U}\left(M_{n}^{n+1}, D\right)$. Since $H: Z \rightarrow\left(\mathcal{H}_{U}\left(M_{n}^{n+1}, D\right), \mathcal{T}\right)$ is a closed map, the set $X_{s}=\left\{H_{z}: z \in \mathcal{E}_{s}\right\}$ is closed with respect to $\mathcal{T}$ for each $s \in T$. We define $\left(E_{s}\right)_{s \in T}$ as follows:

$$
E_{\lambda}=X_{\lambda} \circ J, \quad E_{s}=X_{q_{1} \ldots q_{k}} \circ f_{q_{0}} \quad \text { if } s=q_{0} \ldots q_{k} \in T \backslash\{\lambda\} .
$$

Note that if $f \in \mathcal{H}_{U}\left(M_{n}^{n+1}, D\right)$ then the map $h \mapsto h \circ f$ is a homeomorphism of $\left(\mathcal{H}_{U}\left(M_{n}^{n+1}, D\right), \mathcal{T}\right)$ as well as of $\mathcal{H}_{U}\left(M_{n}^{n+1}, D\right)$. So every $E_{s}$ is closed with respect to $\mathcal{T}$ provided $s \neq \lambda$.

It remains to show that $\left(E_{s}\right)_{s \in T}$ satisfies conditions $\left(1^{\prime}\right)-\left(4^{\prime}\right)$ of Definition 2.20. Since $X_{\lambda} \neq \emptyset$, the set $E_{\lambda}$, just as $J$, is dense in $\mathcal{H}_{U}\left(M_{n}^{n+1}, D\right)$. The other part of condition $\left(1^{\prime}\right)$ follows with the same ease. Since $H: \mathcal{E} \rightarrow$ $\mathcal{H}_{U}\left(M_{n}^{n+1}, D\right)$ is an imbedding, condition $\left(3^{\prime}\right)$ is satisfied. Now let $W$ be an arbitrary set in $\mathcal{H}_{U}\left(M_{n}^{n+1}, D\right)$ such that $\operatorname{diam}(W)<\rho\left(-\infty_{0}, \infty_{0}\right)$. We show that $W$ works for condition $\left(2^{\prime}\right)$ as well as for $\left(4^{\prime}\right)$. Let $\sigma=q_{0} q_{1} \ldots \in[T]$ be such that $E_{\sigma \nmid k} \cap W \neq \emptyset$ for each $k \in \omega$. Putting $\tau=q_{1} q_{2} \ldots \in[T]$ we have $X_{\tau \uparrow k} \cap\left(W \circ f_{q_{0}}^{-1}\right) \neq \emptyset$ for each $k \in \omega$. Since $\hat{\rho}$ is right invariant it follows that

$$
\operatorname{diam}_{\hat{\rho}}\left(W \circ f_{q_{0}}^{-1}\right)<\rho\left(-\infty_{0}, \infty_{0}\right)
$$

and hence $F=\left\{z \in \mathcal{E}: H_{z} \in W \circ f_{q_{0}}^{-1}\right\}$ is bounded by Claim 3.5. Thus $F$ is an anchor for $\left(\mathcal{E}_{s}\right)_{s \in T}$ in $Z$ and obviously $\mathcal{E}_{\tau \uparrow k} \cap F \neq \emptyset$ for each $k \in \omega$. Thus $\mathcal{E}_{\tau\lceil 0}, \mathcal{E}_{\tau \uparrow 1}, \ldots$ converges to an element $z$ in $Z$. Then $X_{\tau \uparrow 0}, X_{\tau \uparrow 1}, \ldots$ converges to $H_{z}$ and $E_{\sigma\lceil 0}, E_{\sigma\lceil 1}, \ldots$ converges to $H_{z} \circ f_{q_{0}}$, both with respect to $\mathcal{T}$. Thus condition $\left(2^{\prime}\right)$ is satisfied. Now let $C$ be a nonempty clopen subset of some $E_{s}$ such that $C \subset W$. We may assume that $|s| \geq 1$ and we put $q=s\lceil 1$ and $q^{\frown} t=s$. So $\operatorname{diam}_{\hat{\rho}}\left(C \circ f_{q}^{-1}\right)<\rho\left(-\infty_{0}, \infty_{0}\right)$ and $C \circ f_{q}^{-1}$ is a nonempty clopen subset of $X_{t}$. This means that $\left\{z \in \mathcal{E}: H_{z} \in C \circ f_{q}^{-1}\right\}$ is a nonempty, clopen, bounded subset of $\mathcal{E}_{t}$. As mentioned above, this contradicts Corollary 2.15, so we conclude that $\left(4^{\prime}\right)$ is satisfied and $\mathcal{H}_{U}\left(M_{n}^{n+1}, D\right) \in \mathrm{E}^{\prime}$. Now apply Theorem 2.21 to see that $\mathcal{H}_{U}\left(M_{n}^{n+1}, D\right)$ is homeomorphic to $\mathfrak{E}$.

CASE (ii). In this case $D \cap \partial U_{i} \cap O$ is dense in $\partial U_{i} \cap O$ for every $i$ and the interior points of $M_{n}^{n+1}$ contained in $D \cap O$ are dense in $O$. We use the same method as in case (i). Take $k \in \omega$. By Proposition 3.4 we choose the imbedding $G^{k}$ in (1) again such that the sets $A_{k}$ and $\mathbb{R}_{k}$ both consist 
of interior points of $M_{n}^{n+1}$. Noting that $\mathbb{R}_{k}$ is a nowhere dense subset of $M_{n}^{n+1}$ we can find a countable dense subset $D_{k}$ of $V_{k}$ such that $A_{k} \subset D_{k}$, $D_{k} \cap \mathbb{R}_{k}=\emptyset, D_{k} \cap \partial U_{i}$ is dense in $\partial U_{i} \cap V_{k}$ for every $i$ with $\partial U_{i} \cap V_{k} \neq \emptyset$, and the interior points of $M_{n}^{n+1}$ in $D_{k}$ are also dense in $V_{k}$. Furthermore, we may assume that $G_{z}^{k}\left(D_{k}\right)=D_{k}$ for each $z \in P$, since $P$ is countable and $G_{z}^{k}\left(\mathbb{R}_{k}\right)=\mathbb{R}_{k}$ for all $z \in E_{4}$. It follows from Lemma 2.9 that we may assume that $D$ has the properties in $(2)$. We continue in precisely the same way as in case (i) to conclude that $\mathcal{H}_{U}\left(M_{n}^{n+1}, D\right) \in \mathrm{E}^{\prime}$ and hence $\mathcal{H}_{U}\left(M_{n}^{n+1}, D\right)$ is homeomorphic to $\mathfrak{E}$ according to Theorem 2.21,

CASE (iii). In this case $D \cap \partial U_{i} \cap O$ is dense in $\partial U_{i} \cap O$ for every $i$ and $D \cap O$ contains no interior points of $M_{n}^{n+1}$. Again, we want $D$ to have the properties (2) for appropriate sets $D_{k}$ so that in the same way as in case (i) (and (ii)) we can conclude that $\mathcal{H}_{U}\left(M_{n}^{n+1}, D\right)$ is homeomorphic to $\mathfrak{E}$. We have to treat the cases $n=1$ and $n>1$ separately.

First we consider the case $n=1$. We want $D \cap V_{0}=D_{0}$, with $D_{0}$ a countable dense subset of $V_{0}$ with $A_{0} \subset D_{0}$ and $D_{0} \cap \mathbb{R}_{0}=\emptyset$. Since $D$ only contains boundary points of $M_{1}^{2}$, we want $D_{0}$ to consist of boundary points of $M_{1}^{2}$. Furthermore, since we are aiming towards Remark 2.10 again, we also want $D_{0}$ to be dense in $\partial U_{i} \cap V_{0}$ for every $i$ with $\partial U_{i} \cap V_{0} \neq \emptyset$. This means that $\mathbb{R}_{0}$ cannot be contained in the boundary of some component $U_{i}$ of the complement of $M_{1}^{2}$. Therefore, we choose $G^{0}$ in $(1)$ such that $A_{0}$ consists of boundary points of $M_{1}^{2}$, and $\mathbb{R}_{0}$ consists of interior points of $M_{1}^{2}$. This is possible according to Proposition 3.4. It is then clear that we can find a set $D_{0}$ as required and by Remark 2.10 we may indeed conclude that $D \cap V_{0}=D_{0}$.

Now take $k \in \mathbb{N}$. Just as in (2) we want $D \cap V_{k}=\mathbb{Q}_{4}^{k} \cup D_{k}$, where $D_{k}$ is a countable dense subset of $V_{k}$ with $D_{k} \cap \mathbb{R}_{k}=\emptyset$ and $A_{k} \subset D_{k}$. Since $D$ consists entirely of boundary points of $M_{1}^{2}$, we choose $G^{k}$ in (1) such that both $A_{k}$ and $\mathbb{R}_{k}$ contain only boundary points of $M_{1}^{2}$. This can be done according to Proposition 3.4. Suppose that $\mathbb{R}_{k} \subset \partial U_{i_{k}}$ for some component $U_{i_{k}}$ of the complement of $M_{1}^{2}$. Noting that $\mathbb{R}_{k}$ is a nowhere dense subset of $M_{1}^{2}$ we can choose $D_{k}$ so that it consists of boundary points of $M_{1}^{2}$, it is dense in $\partial U_{i} \cap V_{k}$ for every $i \in \omega \backslash\left\{i_{k}\right\}$ with $\partial U_{i} \cap V_{k} \neq \emptyset$, and it is dense in $\left(\partial U_{i_{k}} \backslash \mathbb{R}_{k}\right) \cap V_{k}$. We see that $D_{k} \cup \mathbb{Q}_{4}^{k}$ is a countable dense subset of $V_{k}$, entirely consisting of boundary points of $M_{1}^{2}$, that is dense in $\partial U_{i} \cap V_{k}$ for every $i$ with $\partial U_{i} \cap V_{k} \neq \emptyset$. It then follows from Remark 2.10 that we may assume that indeed $D \cap V_{k}=\mathbb{Q}_{4}^{k} \cup D_{k}$.

We conclude that we may assume that $D$ satisfies (2). As before, we may assume that $G_{z}^{k}\left(D_{k}\right)=D_{k}$ for all $k \in \omega$ and $z \in P$, so we can continue in the same way as in case (i) to conclude that $\mathcal{H}_{U}\left(M_{n}^{n+1}, D\right)$ is homeomorphic to $\mathfrak{E}$. 
Now consider the case $n \in \mathbb{N} \backslash\{1,3\}$. This is easier than the onedimensional case. Take $k \in \omega$. Using Proposition 3.4 we choose the imbed$\operatorname{ding} G^{k}$ in (1) such that both the sets $A_{k}$ and $\mathbb{R}_{k}$ consist of boundary points of $M_{n}^{n+1}$. Note that if $\mathbb{R}_{k} \subset \partial U_{i_{k}}$ then $\mathbb{R}_{k}$ is, in contrast to the case $n=1$, nowhere dense in $\partial U_{i_{k}}$. This means that we can find a countable dense subset $D_{k}$ of $V_{k}$, consisting of boundary points of $M_{n}^{n+1}$, such that $A_{k} \subset D_{k}$, $D_{k} \cap \mathbb{R}_{k}=\emptyset$ and $D_{k} \cap \partial U_{i}$ is dense in $\partial U_{i} \cap V_{k}$ for all $i$ such that $V_{k} \cap \partial U_{i} \neq \emptyset$. From Remark 2.10 it follows that we may assume that $D \cap V_{0}=D_{0}$ if $k=0$ and $D \cap V_{k}=\mathbb{Q}_{4}^{k} \cup D_{k}$ if $k \in \mathbb{N}$, so we may assume (2). Again, without loss of generality we have $G_{z}^{k}\left(D_{k}\right)=D_{k}$ for all $k \in \omega$ and $z \in P$, so the same reasoning as in case (i) shows that $\mathcal{H}_{U}\left(M_{n}^{n+1}, D\right)$ is homeomorphic to $\mathfrak{E}$.

In analogy to [5, Theorem 10.4] and [5, Remark 10.7] we can adapt the proof of Theorem 3.1 to produce the following slight generalization.

TheOREM 3.6. Let $X$ be a locally compact space and let $D^{\prime}$ be a countable dense subset of $X$. Suppose that $X$ contains an open subset $O^{\prime}$ that is homeomorphic to an open $O \subset M_{n}^{n+1}$ for some $n \in \mathbb{N} \backslash\{3\}$, such that $D^{\prime} \cap O^{\prime}$ corresponds to a countable dense subset $D$ of $O$ that satisfies the conditions of Theorem 3.1. Then $\mathcal{H}_{U}\left(X, D^{\prime}\right)$ is homeomorphic to $\mathfrak{E}$ for every open set $U$ that contains $O^{\prime}$.

Acknowledgments. The research of the second author was supported by grant 613.000 .432 of the Netherlands Organisation for Scientific Research (NWO).

\section{References}

[1] R. Bennett, Countable dense homogeneous spaces, Fund. Math. 74 (1972), 189-194.

[2] J. W. Cannon, A positional characterization of the $(n-1)$-dimensional Sierpinski curve in $S^{n}(n \neq 4)$, ibid. 79 (1973), 107-112.

[3] J. J. Dijkstra, A criterion for Erdős spaces, Proc. Edinburgh Math. Soc. (2) 48 (2005), 595-601.

[4] -, On homeomorphism groups of Menger continua, Trans. Amer. Math. Soc. 357 (2005), 2665-2679.

[5] J. J. Dijkstra and J. van Mill, Erdös space and homeomorphism groups of manifolds, Mem. Amer. Math. Soc., in press.

[6] R. Engelking, Dimension Theory, North-Holland, Amsterdam, 1978.

[7] —, General Topology, Sigma Ser. Pure Math. 6, Heldermann, Berlin, 1989.

[8] J. van Mill, The Infinite-Dimensional Topology of Function Spaces, North-Holland, Amsterdam, 2001.

[9] J. R. Munkres, Elements of Algebraic Topology, Addison-Wesley, Menlo Park, CA, 1984.

[10] L. G. Oversteegen and E. D. Tymchatyn, On the dimension of certain totally disconnected spaces, Proc. Amer. Math. Soc. 122 (1994), 885-891. 
[11] G. T. Whyburn, Topological characterization of the Sierpiniski curve, Fund. Math. 45 (1958), 320-324.

Jan J. Dijkstra, Dave Visser

Faculteit der Exacte Wetenschappen/Afdeling Wiskunde

Vrije Universiteit Amsterdam

De Boelelaan 1081

$1081 \mathrm{HV}$ Amsterdam

The Netherlands

E-mail: dijkstra@few.vu.nl daveviss@gmail.com

Received 11 November 2008;

in revised form 4 October 2009 